\title{
Beta-blockers are the old BLACK
}

\author{
W. Scott Beattie, MD, PhD • Gregory M. T. Hare, MD, PhD
}

Received: 9 June 2014/ Accepted: 17 June 2014/ Published online: 30 July 2014

(c) Canadian Anesthesiologists' Society 2014

Beta-blockers, as their raison d'être, were developed with the intent to attenuate the heart's responses to stress. Sir James Black, a Scottish pharmacologist, held the view that antagonizing the catecholamine-mediated augmenting effects on heart rate and myocardial contractility would relieve the pain of angina pectoris through a decrease in the heart's requirement for oxygen. ${ }^{1}$ His theories led to the discovery of propranolol (and later atenolol) and, in turn, a whole pharmacology industry dedicated to the synthesis and marketing of a variety of adrenergic antagonists that favorably altered the outcomes of numerous cardiac-related maladies. ${ }^{2-4}$ To this day, beta-blockers remain effective agents that reduce all measure of cardiac events. ${ }^{2-4}$ In 1988, Sir James was awarded the Nobel Prize for his efforts. ${ }^{\text {A }}$

Unfortunately for the field of perioperative medicine, we now have overwhelming evidence that this ability to attenuate cardiac responses to stress also leads to unwanted effects distant from the heart, which themselves are unmasked by the stresses of surgery. ${ }^{5,6}$ It is now well recognized that metoprolol increases the rate of

\footnotetext{
A The Nobel Prize also recognized Dr. Black's role in the development of cimetidine.
}

Reprints will not be available from the authors.

W. S. Beattie, MD, PhD - G. M. T. Hare, MD, PhD

Department of Anesthesia at the University Health Network, St. Michael's Hospital, Toronto, ON, Canada

W. S. Beattie, MD, $\mathrm{PhD}$ - G. M. T. Hare, MD, PhD Department of Anesthesia, The Peter Munk Cardiac Centre, The University of Toronto, Toronto, ON, Canada

W. S. Beattie, MD, PhD ( $\square)$

Department of Anesthesiology, Toronto General Hospital, 200

Elizabeth Street, Toronto, ON M5G 2C4, Canada

e-mail: Scott.beattie@unh.ca perioperative strokes. ${ }^{7}$ In this issue of the Journal, Dr. Florea et $a l .{ }^{8}$ from Queen's University add to this growing list of undesirable peripheral effects by reporting an increased incidence of cerebral ischemia in patients on beta-blockers (i.e., predominately metoprolol) after arterial cross-clamping during elective carotid endarterectomy. Their institution uses an intraoperative process of care that includes electroencephalogram (EEG) monitoring to detect cerebral ischemia during all procedures. The process further dictates that a shunt will be employed for patients demonstrating cerebral ischemia. Using this database of 523 consecutive patients over a ten-year period, they found $69(13.2 \%)$ patients with intraoperative EEG evidence of cerebral ischemia. Using a case-controlled design where patients with ischemia are matched to controls in a one-toone ratio, Florea et al. show that patients on beta-blockers were associated with more than a twofold increase in the incidence of ischemia and therefore shunt utilization. The increased incidence of ischemia is both biologically plausible and in keeping with three recent reports all showing that chronically beta-blocked patients have increased rates of perioperative delirium ${ }^{9}$ and stroke. ${ }^{10,11}$ The results are also similar to findings in the published prospective randomized beta-blocker trials. ${ }^{5}$

All retrospective and observational trials have limitations, and these authors are commended for enumerating the shortcomings in the present report. The most obvious limitation is the retrospective nature of this chart review that renders it prone to residual confounding. We are particularly concerned, in this instance, about the sample size and the low number of outcomes; both of these factors limit the ability to conduct adequate and stable statistical modelling. In this analysis, the control cohort was generated from patients without cerebral ischemia matched by year of surgery and contralateral carotid 
occlusion. The balance between the two cohorts (their Table 1) was assessed using bivariate null hypothesis tests. This statistical methodology (i.e., $P$ value) used in evaluating balance in artificial matching scenarios is prone to error since it relies on sample size. ${ }^{12}$ Alternatively, the standardized difference compares the difference in means, is not influenced by sample size, and allows comparison of the balance of variables measured in different units. There is no set criterion as to which threshold of the standardized difference would indicate important imbalance, but a difference that is more than 0.1 is now generally accepted to indicate important differences between the intervention and control groups. ${ }^{13}$ As well, in the present analysis, hypertension, diabetes, history of stroke, and contralateral carotid disease, all of which are associated with postoperative stroke, ${ }^{14,15}$ are each poorly matched on the basis of the calculated standardized differences. Thus, as the authors point out, the possibility that the increased incidence of cerebral ischemia afforded to beta-blockade is due to residual confounding cannot be ruled out. Despite these limitations, the fact remains that every trial (prospective randomized controlled trials and observational studies) has found metoprolol to increase perioperative strokes. Each of these analyses has also shown a significant association in patients with

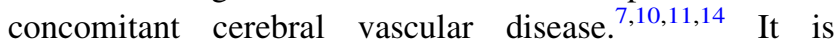
unclear from any of these studies if the increased incidence in stroke in beta-blocked patients is seen in patients without cerebral vascular disease. In our opinion, use of perioperative metoprolol in patients with cerebral vascular disease should be carefully considered with an awareness of the possible clinical outcomes.

The authors also raise a critical safety issue - the withdrawal of beta-blockade. Remembering that betablocker therapy is often chronically prescribed to patients with a higher cardiac risk in response to decades-old Grade 1 evidence, ${ }^{16-18}$ the question of beta-blocker withdrawal is a reasonable issue to explore. Inevitably, some of the patients on recommended beta-blocker therapy will require surgery, and the question of withdrawal is certainly justified on the basis of the accumulated evidence. If beta-adrenergic antagonism increases mortality and stroke, why should we continue their use in the perioperative setting? We think that changing established cardiovascular therapy before surgery would be premature since the present evidence is insufficient to address the balance of risks and benefits.

The present analysis was designed to assess only the relationship between beta-blockade and ischemic EEG changes after carotid artery cross-clamping. The ischemic changes detected by EEG in this present study are surrogate outcomes that do not assess the relationship between beta-blockade and stroke, myocardial infarction (MI), or mortality. These specific outcomes cannot be assessed using the present methodology. Assessment of death and MI requires a sample size in the thousands. ${ }^{19}$ Myocardial infarction can be determined accurately only with routine sampling of cardiac biomarkers. ${ }^{20}$ Sporadic sampling of cardiac biomarkers, as was conducted in this study, can underestimate the incidence of MI by up to threefold. $^{21}$ Finally, recently identified factors, those of chronicity of dosing and beta-1 selectivity receptor antagonism, raise questions about the generalizability of many of the safety issues in the POISE-I trial. ${ }^{7}$

Two aspects of the beta-blocker studies conducted to date deserve closer scrutiny: 1) When is the optimum time to start beta-blockers (i.e., chronic $v s$ acute use)? 2) Is there a superior type of beta-blocker (i.e., receptor selectivity and pharmacokinetic characteristics)?

The distinction between acute and chronic beta-blockade is highly topical. ${ }^{22,23}$ The randomized trials predominately excluded chronically dosed patients, which led to the exclusion of many higher risk patients, thus limiting the generalizability. In contrast to the randomized trials, a number of well-conducted studies with observational data indicate that chronic beta-blockade in high-risk noncardiac surgical patients is associated with reduced mortality. ${ }^{24-26} \mathrm{Of}$ particular importance, an evaluation of Ontario's drug benefit plan regarding patients over $65 \mathrm{yr}$ having surgery indicated that starting beta-blockers within seven days of surgery increased the incidence of MI and death when compared with starting patients on beta-blockers more than 30 days preoperatively. ${ }^{27}$ Interestingly, and contrary to MI and death, the incidence of stroke was the same; chronicity of dosage did not affect outcome. Nevertheless, the incidence of stroke in these beta-blocked (acute or chronic) patients is stable at $0.5 \%$, a rate that is twice that in patients naive to these agents.

Metoprolol appears to be an inferior beta-antagonist. The role of $\beta 1$-selective agents and the effects of the cytochrome P-450 isoenzyme, CYP2D6, have been shown to have important effects on many cardiovascular outcomes (stroke and MI) in general medicine. ${ }^{28,29} \mathrm{~A}$ meta-analysis of the randomized studies of perioperative beta-blockers has suggested that metoprolol results in more adverse outcomes than either atenolol or bisoprolol. ${ }^{30}$ This analysis highlighted the importance of two proposed hypotheses that could explain the increased adversity seen within the metoprolol studies. First, the benefit of beta-blockers is reduced for blockers metabolized by the CYP2D6 isoenzyme. This reduction could occur because of individual variations in CYP2D6 activity secondary to either genetics or drug-to-drug interactions. The second hypothesis relates to the relative ratio of $\beta-1$ to $\beta-2$. Drugs with poor $\beta-1$ selectivity may have deleterious effects on vital organ vascular regulation, since the residual $\beta-2$ antagonism may interfere with compensatory increases in 
oxygen deliver during periods of stress. The hypothesis has biologic plausibility, since translational animal studies show severe impairment of cerebral oxygen delivery with metoprolol, with weak selectivity but relatively preserved cerebral perfusion with the highly beta- 1 selective antagonist, nebivolol. ${ }^{31}$ The recent large observational studies universally show that metoprolol is inferior compared with other more selective beta-blockers. Whether this is due to selectivity or kinetics (or both) is irrelevant. ${ }^{10,11,25,32,33} \mathrm{We}$ are therefore presented with an incredibly powerful signal, the randomized trials, translational animal studies, and observational studies all show the same result; metoprolol is an inferior agent, and employing more beta-1 selective agents may improve all cardiovascular outcomes.

The efficacy of beta-blockers has never been the issue; it is one of safety. The POISE- $\mathrm{I}^{7}$ and the myriad of metaanalyses that have followed make clear that perioperative beta-blockade reduces perioperative MI. Now with the publication of POISE-II, ${ }^{\mathrm{B}, 34-36}$ beta-blockers remain the only method that has been shown to reduce postoperative MI reliably. Thus, if the safety aspects of perioperative beta-blockade could be remedied, selective perioperative beta-blockade would have enormous utility.

Dr. Florea and her colleagues have shown an important new relationship that is congruent with previous findings. These new findings, however, should not tip the balance away from using beta-blockers in surgical patients with higher cardiac risk. The findings are limited to carotid surgical patients who, by definition, have cerebral vascular disease. The findings greatly support the idea that use of metoprolol in this population may lead to an increased risk for inadequate cerebral perfusion. Based on this and many other lines of evidence, the further use of metoprolol in this population will require strong justification. Nevertheless, beta-blockers remain the only proven mode of attenuating postoperative MI, a leading and often life-threatening adverse outcome. We are now obliged to test the new and promising hypotheses, which, on the basis of many independently conducted observational trials, have the potential to reduce the adverse events associated with perioperative metoprolol. We invite all to join us on our quest.

\section{Les bêta-bloqueurs ne sont pas tout noir ou tout blanc}

Les bêta-bloqueurs ont été développés spécifiquement dans le but d'atténuer les réponses du cœur au stress. Sir James

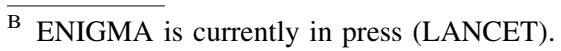

Black, un pharmacologue écossais, pensait qu'en antagonisant les effets médiés par les catécholamines d'accélération de la fréquence cardiaque et d'accroissement de la contractilité myocardique, cela soulagerait la douleur de l'angine de poitrine par la diminution des besoins du cœur en oxygène. ${ }^{1}$ Ses théories ont conduit à la découverte du propranolol (et, plus tard, de l'aténolol); une branche complète de l'industrie pharmaceutique s'est à son tour dédiée à la synthèse et à la commercialisation de différents antagonistes adrénergiques qui modifiaient de façon favorable l'évolution de nombreuses maladies en rapport avec le cœur. $^{2-4}$ Jusqu'à ce jour, les bêta-bloqueurs restent des agents efficaces pour réduire tous les critères des événements cardiaques. ${ }^{2-4}$ Les efforts de Sir James ont été couronnés par la remise d'un prix Nobel en 1988. ${ }^{\text {A }}$

Malencontreusement, pour le domaine de la médecine périopératoire, nous disposons aujourd'hui d'une volumineuse masse de données probantes montrant que cette capacité à atténuer les réponses cardiaques au stress mène également à des effets indésirables à distance du cœur, qui sont eux-mêmes démasqués par le stress de la chirurgie. ${ }^{5,6} \mathrm{Il}$ est maintenant bien établi que le métoprolol augmente la fréquence des AVC périopératoires. ${ }^{7}$ Dans ce numéro du Journal, la docteure Florea et coll. ${ }^{8}$ de l'université Queens allongent encore cette liste grandissante d'effets indésirables périphériques en montrant une plus grande incidence d'ischémie cérébrale chez les patients qui reçoivent des bêta-bloqueurs (principalement du métoprolol) après clampage artériel pour une endartériectomie carotidienne programmée. Cette institution suit une procédure de soins peropératoire qui inclut une surveillance électroencéphalographique (EEG) au cours de toutes les interventions pour la détection d'une ischémie cérébrale. En outre, la procédure oblige à utiliser une dérivation chez les patients chez lesquels une ischémie cérébrale est mise en évidence. En analysant cette base de données de 523 patients consécutifs sur une période de 10 ans, les auteurs ont trouvé $69(13,2 \%)$ patients avec des données probantes d'ischémie cérébrale sur l'EEG peropératoire. Utilisant une conception cas-témoin, dans lequel les patients atteints d'ischémie ont été appariés à des témoins dans un rapport de 1 à 1, Florea et coll. ont montré que les patients recevant des bêta-bloqueurs étaient associés à une augmentation de plus deux fois de l'incidence de l'ischémie (et par conséquent le recours à une dérivation). L'augmentation de l'incidence de l'ischémie est à la fois biologiquement plausible et en phase avec trois publications récentes qui ont toutes montré que les patients recevant de façon chronique des bêta-bloqueurs avaient des taux augmentés de délirium ${ }^{9}$ et $\mathrm{d}^{\prime} \mathrm{AVC}^{10,11}$ peropératoires. Ces résultats sont également semblables aux constatations des essais randomisés prospectifs publiés sur les bêta-bloqueurs. ${ }^{5}$ 
Tous les essais rétrospectifs et observationnels ont des limites et ces auteurs doivent être félicités pour les avoir énumérés dans le présent article. La plus manifeste est la nature rétrospective de cette analyse de dossiers qui la rend sujette à des facteurs confondants résiduels. Nous sommes particulièrement concernés dans le cas présent par la taille de l'échantillon et le faible nombre de résultats; ces deux facteurs limitent la capacité à réaliser une modélisation statistique stable et adéquate. Dans cette analyse, la cohorte témoin a été créée à partir de patients sans ischémie cérébrale, appariés par année de la chirurgie et occlusion de la carotide controlatérale. L'équilibre entre leurs deux cohortes (leur tableau 1) a été évalué en testant l'hypothèse nulle bifactorielle. Cette méthodologie statistique (c'est-à-dire la valeur de $P$ ) utilisée pour l'évaluation de l'équilibre dans des scénarios d'appariement artificiels est encline à l'erreur dans la mesure où elle repose sur la taille de l'échantillon. ${ }^{12}$ Sinon, la différence standardisée compare la différence entre les moyennes et n'est pas influencée par la taille de l'échantillon; elle permet également la comparaison de l'équilibre de variables mesurées avec des unités différentes. Il n'y a pas de critère établi pour dire quel seuil de différence standardisée indique un déséquilibre important, mais une différence supérieure à 0,1 est maintenant généralement acceptée pour signaler l'existence de différences importantes entre le groupe interventionnel et le groupe contrôle. ${ }^{13}$ Dans la présente analyse, une hypertension, un diabète, des antécédents d'AVC et d'atteinte carotidienne controlatérale (qui sont tous associés à des AVC postopératoires) ${ }^{14,15}$ sont aussi, chacun, faiblement appariés sur la base des différences standardisées calculées. Ainsi, comme le font remarquer les auteurs, la possibilité qu'une incidence accrue d'ischémie cérébrale attribuée au blocage bêta soit due à un facteur confondant résiduel ne peut pas être exclue. Malgré ces Limites, un fait demeure: chaque étude - les études randomisées prospectives et contrôlées comme les études observationnelles - a constaté que le métoprolol augmente le taux d'ACV périopératoires. Chacune de ces analyses a également montré une association significative chez les patients qui avaient une maladie vasculaire cérébrale concomitante. ${ }^{7,10,11,14}$ Aucune de ces études ne permet de conclure formellement que la plus forte incidence d'AVC chez des patients ayant reçu des bêta-bloqueurs est aussi constatée chez des patients sans maladie vasculaire cérébrale. Selon nous, l'utilisation périopératoire de métoprolol chez des patients ayant une maladie vasculaire cérébrale doit être envisagée avec précaution en ayant conscience des conséquences cliniques possibles.

Les auteurs soulèvent également un problème critique d'innocuité: le retrait des bêta-bloqueurs. En se rappelant que le traitement par bêta-bloqueurs est souvent prescrit chroniquement chez des patients à haut risque cardiaque en réponse à des décennies de preuves de niveau ${ }^{1,16}$ il est raisonnable de poser la question sur le retrait des bêta-bloqueurs. Il est inévitable que certains patients qui reçoivent une thérapie recommandée par bêta-bloqueurs aient besoin un jour d'une intervention chirurgicale. La question du retrait est certainement justifiée sur la base des données probantes accumulées. Si l'antagonisme bêta adrénergique augmente la mortalité et les AVC, pourquoi devrions-nous continuer à les utiliser en périopératoire? Nous pensons que de modifier le traitement cardiovasculaire reconnu avant une intervention chirurgicale serait prématuré, car la preuve actuelle est insuffisante pour répondre à la question de l'équilibre avantages-risques.

Cette analyse a été conçue pour évaluer uniquement les rapports entre le traitement par bêta-bloqueurs et les modifications ischémiques à l'EEG après clampage de l'artère carotide. Les modifications ischémiques détectées à l'EEG, observées dans la présente étude, sont un critère d'évaluation de substitution qui n'évalue pas les relations entre le blocage bêta et les AVC, l'infarctus du myocarde (IM) ou la mortalité. Ces critères d'évaluation spécifiques ne peuvent pas être évalués en utilisant cette méthodologie. L'évaluation des décès et des IM nécessite un échantillon dont la taille se situe dans les milliers. ${ }^{19}$ Un infarctus du myocarde ne peut être déterminé avec exactitude qu'avec des prélèvements réguliers de biomarqueurs cardiaques. ${ }^{20}$ Un échantillonnage occasionnel de biomarqueur cardiaque, comme ce fut le cas dans cette étude, risque de sous-estimer l'incidence des IM d'un facteur pouvant aller jusqu'à trois fois. ${ }^{21}$ Enfin, des facteurs récemment identifiés, ceux de la chronicité de la dose et le caractère sélectif de l'antagonisme des récepteurs bêta-1, soulèvent des questions sur la possibilité de généraliser un grand nombre de questions de sécurité de l'étude POISE-I. ${ }^{7}$

Deux aspects des études concernant les bêta-bloqueurs menées à ce jour méritent d'être examinés de plus près. 1) Quel est le meilleur moment pour commencer un traitement par bêta-bloqueurs? (c'est-à-dire utilisation chronique ou en phase aiguë) et 2) existe-t-il un type supérieur de bêta-bloqueur? (c'est-à-dire avec une affinité pour le récepteur et des caractéristiques pharmacocinétiques données).

La distinction entre un blocage aigu ou chronique par bêta-bloqueurs est d'une grande actualité. ${ }^{22,23}$ Les études randomisées ont exclu, principalement, les patients qui étaient traités de façon chronique, conduisant à l'exclusion de nombreux patients à haut risque, limitant du même coup la possibilité de généralisation. En revanche et contrairement aux études randomisées, un certain nombre d'études bien menées avec des données observationnelles indiquent qu'un blocage chronique bêta chez des patients chirurgicaux non cardiaques à risque élevé était associé à une baisse de la 
mortalité. ${ }^{24-26}$ Particulièrement intéressant, le plan ontarien d'évaluation de bénéfice des drogues chez les patients âgés de plus de 65 ans subissant une intervention chirurgicale, a indiqué que le commencement de bêta-bloqueurs dans les 7 jours précédant la chirurgie augmentait l'incidence des IM et des décès, comparativement aux patients ayant commencé le traitement plus de 30 jours avant l'intervention. ${ }^{27}$ Il est intéressant de noter que, contrairement à l'incidence des IM et des décès, celle des AVC est restée la même; le caractère chronique de l'administration n'a pas eu d'influence sur l'évolution. Cependant, l'incidence des AVC dans cette population de patients ayant un blocage bêta (aigu ou chronique) est stable à $0,5 \%$, un taux deux fois plus élevé que chez les patients naïfs pour ce type de drogues.

Le metoprolol semble être un bêta-antagoniste plus faible. Le rôle des agents $\beta 1$-sélectifs et les effets de l'isoenzyme CYP2D6 du cytochrome P450 ont été démontrés: ils ont des effets importants sur de nombreux critères d'évaluation cardiovasculaires (AVC et IM) et médicaux, en général. ${ }^{28,29}$ Une méta-analyse des études randomisées périopératoires avec les bêta-bloqueurs a suggéré que le métoprolol entraînait plus d'événements indésirables que l'aténolol ou le bisoprolol. ${ }^{30}$ Cette analyse a souligné l'importance des deux hypothèses proposées qui pourraient expliquer les plus mauvais résultats constatés dans les études avec le métoprolol. Tout d'abord, le bénéfice des bêta-bloqueurs est réduit chez les bêta-bloqueurs métabolisés par l'isoenzyme CYP2D6. Cette baisse pourrait être due aux variations individuelles de l'activité du CYP2D6, d'origine génétique ou due à des interactions médicamenteuses. La seconde hypothèse est liée au rapport relatif $\beta-1 / \beta-2$. Les drogues ayant une faible sélectivité $\beta 1$ pourraient avoir des effets délétères sur la régulation vasculaire d'un organe vital, car l'antagonisme $\beta 2$ résiduel pourrait interférer avec les augmentations compensatoires de distribution de l'oxygène au cours des périodes de stress. L'hypothèse est biologiquement plausible considérant que les études translationnelles chez l'animal montrent une altération importante de l'oxygénation cérébrale par le métoprolol, avec une faible sélectivité, mais une perfusion cérébrale relativement préservée avec un antagoniste $\beta 1$ hautement sélectif comme le nébivolol. ${ }^{31}$ Les grandes études observationnelles récentes montrent indiscutablement que le métoprolol est inférieur à d'autres bêta-bloqueurs plus sélectifs, et que cela soit dû à la sélectivité ou à la cinétique, ou les deux, n'a pas d'importance..$^{10,11,25,32,33}$ Nous trouvons donc ici un signal incroyablement puissant puisque les études randomisées, les études translationnelles chez l'animal et les études observationnelles, montrent - toutes - le même résultat. Le métoprolol est un agent inférieur et l'utilisation de molécules $\beta 1$-sélectives pourrait améliorer tous les critères d'évaluation cardiovasculaires.
L'efficacité des bêta-bloqueurs n'a jamais été le problème; c'est une question d'innocuité. POISE- $\mathrm{I}^{7}$ et la myriade de méta-analyses qui ont suivi montrent clairement que le blocage bêta périopératoire diminue le nombre d'IM périopératoires. Maintenant, avec la publication de POISE-II ${ }^{34,35}$ et d'ENIGMA-II (bien que ses résultats ne soient pas encore connus), ${ }^{\mathrm{B}, 36}$ les bêta-bloqueurs restent la seule méthode ayant démontré qu'elle réduisait de façon fiable les IM postopératoires. Ainsi, si les aspects d'innocuité du blocage périopératoire bêta pouvaient être résolus, un blocage bêta sélectif périopératoire serait d'une utilité considérable.

La Dre Florea et ses collègues ont montré une importante et nouvelle relation qui va dans le sens des constatations antérieures. Toutefois, cette nouvelle constatation ne devrait pas faire bouger la balance pour éloigner les patients chirurgicaux à plus grand risque cardiaque de l'utilisation des bêta-bloqueurs. Ces constatations sont limitées aux patients subissant une intervention sur la carotide qui, par définition, ont une maladie vasculaire cérébrale. Elles soutiennent aussi largement l'idée selon laquelle, dans cette population, le métoprolol peut entraîner un plus grand risque de perfusion cérébrale inadéquate. Sur cette base ainsi que sur les nombreuses autres lignes de données probantes, la poursuite de l'utilisation du métoprolol dans cette population devra être solidement justifiée. Les bêta-bloqueurs restent néanmoins le seul mode prouvé d'atténuation des IM postopératoires, un événement indésirable fréquent et mettant souvent la vie des patients en jeu. Nous sommes maintenant dans l'obligation de tester de nouvelles hypothèses prometteuses qui, en s'appuyant sur de nombreuses études observationnelles menées de façon indépendante, pourraient diminuer les événements indésirables associés au métoprolol périopératoire. Nous vous invitons tous à nous joindre à cette quête.

Competing interests Dr. Beattie is supported in part by the R. Fraser Elliott Chair in Cardiac Anesthesia Research. Dr. Beattie and Dr. Hare are supported in part by Merit Awards from the Department of Anesthesia at the University of Toronto. Drs. Hare and Beattie have conducted numerous investigations pertaining to the mechanism of beta-blocker-induced injury. Dr. Beattie is the Associate Editor of the Canadian Journal of Anesthesia and Dr. Hare is a member of the same Editorial Board. Dr. Hilary Grocott served as the handling editor for this manuscript; neither Drs. Beattie nor Hare were involved in the review process.

Conflits d'intérêts Le Dr Beattie est soutenu pour une part par la chaire Fraser Elliott de recherche en anesthésiologie cardiaque. Le Dr Hare et le Dr Beattie ont mené de nombreuses investigations ayant trait au mécanisme des lésions induites par les bêta-bloqueurs. Le Dr Beattie et le Dr Hare sont soutenus pour une part par le Prix du mérite du Département d'anesthésie de l'Université de Toronto. Le Dr Beattie est un Associate Editor du Journal canadien d'anesthésie et le Dr Hare est membre du même comité éditorial. Le Dr Hilary Grocott 
a révisé ce manuscrit; ni le Dr Beattie, ni le Dr Hare n'ont été impliqués dans cette procédure de révision.

\section{References}

1. Quirke V. Putting theory into practice: James black, receptor theory and the development of the beta-blockers at ICI, 19581978. Med Hist 2006; 50: 69-92.

2. Freemantle N, Cleland J, Young P, Mason J, Harrison J. Beta blockade after myocardial infarction: systematic review and meta regression analysis. BMJ 1999; 318: 1730-7.

3. Al-Gobari M, El Khatib C, Pillon F, Gueyffier F. Beta-blockers for the prevention of sudden cardiac death in heart failure patients: a meta-analysis of randomized controlled trials. BMC Cardiovasc Disord 2013; 13: 52.

4. Rienstra M, Damman K, Mulder BA, Van Gelder IC, McMurray $J J$, Van Veldhuisen DJ. Beta-blockers and outcome in heart failure and atrial fibrillation: a meta-analysis. JACC Heart Fail 2013; 1: 21-8.

5. Bangalore S, Wetterslev J, Pranesh S, Sawhney S, Gluud C, Messerli FH. Perioperative beta blockers in patients having noncardiac surgery: a meta-analysis. Lancet 2008; 372: 1962-76.

6. Beattie WS, Wijeysundera DN, Karkouti K, et al. Acute surgical anemia influences the cardioprotective effects of beta-blockade: a single-center, propensity-matched cohort study. Anesthesiology 2010; 112: 25-33.

7. Study Group POISE, Devereaux PJ, Yang H, Yusuf S, et al. Effects of extended-release metoprolol succinate in patients undergoing non-cardiac surgery (POISE trial): a randomised controlled trial. Lancet 2008; 371: 1839-47.

8. Florea A, van Vlymen J, Ali S, Day AG, Parlow J. Preoperative beta blocker use associated with cerebral ischemia during carotid endarterectomy. Can J Anesth 2014; 61: this issue. DOI: 10.1007/ s12630-014-0195-9.

9. Katznelson R, Djaiani G, Mitsakakis $N$, et al. Delirium following vascular surgery: Increased incidence with preoperative betablocker administration. Can J Anesth 2009; 56: 793-801.

10. Mashour GA, Sharifpour M, Freundlich RE, et al. Perioperative metoprolol and risk of stroke after noncardiac surgery. Anesthesiology 2013; 119: 1340-6.

11. Ashes C, Judelman S, Wijeysundera DN, et al. Selective beta1antagonism with bisoprolol is associated with fewer postoperative strokes than atenolol or metoprolol: a single-center cohort study of 44,092 consecutive patients. Anesthesiology 2013; 119: 777-87.

12. Austin PC. Balance diagnostics for comparing the distribution of baseline covariates between treatment groups in propensity-score matched samples. Stat Med 2009; 28: 3083-107.

13. Austin PC. An introduction to propensity score methods for reducing the effects of confounding in observational studies. Multivariate Behav Res 2011; 46: 399-424.

14. Mashour GA, Shanks AM, Kheterpal S. Perioperative stroke and associated mortality after noncardiac, nonneurologic surgery. Anesthesiology 2011; 114: 1289-96.

15. Selim M. Perioperative stroke. N Engl J Med 2007; 356: 706-13.

16. Anderson JL, Adams CD, Antman EM, et al.; 2011 Writing Group Members; ACCF/AHA Task Force Members. 2011 ACCF/AHA focused update incorporated into the ACC/AHA 2007 Guidelines for the Management of Patients with Unstable Angina/Non-STElevation Myocardial Infarction: a report of the American College of Cardiology Foundation/American Heart Association Task Force on Practice Guidelines. Circulation 2011; 123: e426-579.
17. Yancy CW, Jessup M, Bozkurt B, et al. 2013 ACCF/AHA Guideline for the Management of Heart Failure: Executive summary: a report of the American College of Cardiology Foundation/American Heart Association Task Force on Practice Guidelines. Circulation 2013; 128: 1810-52.

18. Fihn SD, Gardin JM, Abrams J, et al.; American College of Cardiology Foundation, American Heart Association Task Force on Practice Guidelines, American College of Physicians, American Association for Thoracic Surgery, Preventive Cardiovascular Nurses Association, Society for Cardiovascular Angiography and Interventions, Society of Thoracic Surgeons. 2012 ACCF/AHA/ ACP/AATS/PCNA/SCAI/STS Guideline for the Diagnosis and Management of Patients with Stable Ischemic Heart Disease: a report of the American College of Cardiology Foundation/American Heart Association Task Force on Practice Guidelines, and the American College of Physicians, American Association for Thoracic Surgery, Preventive Cardiovascular Nurses Association, Society for Cardiovascular Angiography and Interventions, and Society of Thoracic Surgeons. J Am Coll Cardiol 2012; 60: e44-164.

19. Davis C, Tait G, Carroll J, Wijeysundera DN, Beattie WS. The Revised Cardiac Risk Index in the new millennium: a singlecentre prospective cohort re-evaluation of the original variables in 9,519 consecutive elective surgical patients. Can J Anesth 2013; 60: 855-63.

20. Thygesen K, Alpert JS, Jaffe AS, et al. Third universal definition of myocardial infarction. Circulation 2012; 126: 2020-35.

21. Beattie WS, Karkouti K, Tait G, et al. Use of clinically based troponin underestimates the cardiac injury in non-cardiac surgery: a single centre cohort study in 51,701 consecutive patients. Can J Anesth 2012; 59: 1013-22.

22. Ellenberger $C$, Tait $G$, Beattie WS. Chronic beta blockade is associated with a better outcome after elective noncardiac surgery than acute beta blockade: a single-center propensity-matched cohort study. Anesthesiology 2011; 114: 817-23.

23. Wallace $A W, A u S$, Cason BA. Association of the pattern of use of perioperative beta-blockade and postoperative mortality. Anesthesiology 2010; 113: 794-805.

24. Andersson C, Merie C, Jorgensen M, et al. Association of betablocker therapy with risks of adverse cardiovascular events and deaths in patients with ischemic heart disease undergoing noncardiac surgery: a Danish nationwide cohort study. JAMA Intern Med 2014; 174: 336-44.

25. London MJ, Hur K, Schwartz GG, Henderson WG. Association of perioperative beta-blockade with mortality and cardiovascular morbidity following major noncardiac surgery. JAMA 2013; 309: 1704-13.

26. Lindenauer PK, Pekow P, Wang K, Mamidi DK, Gutierrez B, Benjamin EM. Perioperative beta-blocker therapy and mortality after major noncardiac surgery. N Engl J Med 2005; 353: 349-61.

27. Wijeysundera DN, Beattie WS, Wijeysundera HC, Yun L, Austin $P C, K o$ DT. Duration of preoperative beta-blockade and outcomes after major elective noncardiac surgery. Can J Cardiol 2014; 30: 217-23.

28. Webb AJ, Fischer U, Rothwell PM. Effects of beta-blocker selectivity on blood pressure variability and stroke: a systematic review. Neurology 2011; 77: 731-7.

29. Rinfret $S$, Abrahamowicz $M, T u J$, et al. A population-based analysis of the class effect of beta-blockers after myocardial infarction. Am Heart J 2007; 153: 224-30.

30. Badgett RG, Lawrence VA, Cohn SL. Variations in pharmacology of beta-blockers may contribute to heterogeneous results in trials of perioperative beta-blockade. Anesthesiology 2010; 113: 585-92.

31. Hu T, Beattie WS, Mazer CD, et al. Treatment with a highly selective beta(1) antagonist causes dose-dependent impairment of 
cerebral perfusion after hemodilution in rats. Anesth Analg 2013; 116: 649-62.

32. Redelmeier D, Scales D, Kopp A. Beta blockers for elective surgery in elderly patients: population based, retrospective cohort study. BMJ 2005; 331: 932.

33. Wallace $A W, A u S$, Cason BA. Perioperative beta-blockade: atenolol is associated with reduced mortality when compared to metoprolol. Anesthesiology 2011; 114: 824-36.

34. Devereaux PJ, Mrkobrada M, Sessler DI, et al.; POISE-2 Investigators. Aspirin in patients undergoing noncardiac surgery. N Engl J Med 2014; 370: 1494-503.
35. Devereaux PJ, Sessler DI, Leslie $K$, et al.; Investigators $P$. Clonidine in patients undergoing noncardiac surgery. N Engl J Med 2014; 370: 1504-13.

36. Myles PS, Leslie K, Peyton $P$, et al. Nitrous oxide and perioperative cardiac morbidity (ENIGMA-II) trial: rationale and design. Am Heart J 2009; 157: 488-94.e1. 